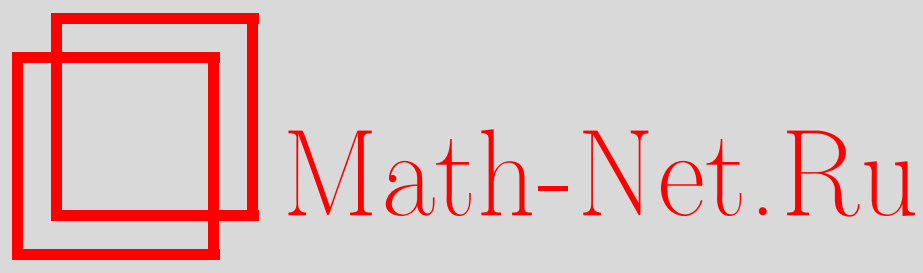

С. С. Марченков, Замкнутые классы трехзначной логики, содержащие существенно многоместные линейные функции, Дискрет. матем., 2015, том 27, выпуск 1, 98 107

DOI: https://doi.org/10.4213/dm1317

Использование Общероссийского математического портала Math-Net.Ru подразумевает, что вы прочитали и согласны с пользовательским соглашением http://www . mathnet.ru/rus/agreement

Параметры загрузки:

IP: 18.234 .156 .22

26 апреля 2023 г., $12: 26: 02$

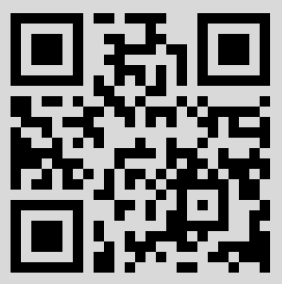




\title{
Замкнутые классы трехзначной логики, содержащие существенно многоместные линейные функции
}

\author{
() 2015 г. С. С. Марченков*
}

Определяются все 23 замкнутых класса трехзначной логики, которые содержат существенно многоместные линейные функции. Устанавливается, что порядок каждого их этих классов не превосходит 3.

Работа выполнена при поддержке Российского фонда фундаментальных исследований, проект 13-01-00958.

Ключевые слова: трехзначная логика, замкнутые классы, линейные функции.

\section{1. Введение}

Одной из распространенных классификаций множества $P_{k}$ функций $k$-значной логики является классификация, базирующаяся на операции суперпозиции. При $k \geqslant 3$ эти классификации континуальны, и «полные» их описания, по-видимому, невозможны. Поэтому исследования решеток $\mathcal{L}_{k}$ замкнутых классов из $P_{k}$ развиваются по пути описания достаточно крупных фрагментов этих решеток. Особый интерес здесь представляют интервалы, а также верхние и нижние конусы, определяемые известными замкнутыми классами. Так, например, при любом $k \geqslant 2$ верхний и нижний конусы, определяемые классом $H_{k}$ всех однородных функций, конечны и имеют конечные базисы. Для нижнего конуса этот результат вытекает из работы [4], для верхнего конуса - из работ $[7,16]$.

Класс $P_{3}$ функций трехзначной логики является «наименьшим» классом, в котором можно найти практически все особенности, характерные для функций многозначных логик. В связи с этим решетка $\mathcal{L}_{3}$ подвергалась исследованиям гораздо в большей степени, нежели решетки $\mathcal{L}_{k}$ при $k>3$. В частности, в решетке $\mathcal{L}_{3}$ определены не только все коатомы (элементы решетки, соответствующие предполным классам в $P_{3}$ ), но и атомы этой решетки [17], а также все элементы, лежащие на «глубине» 2 [18] (которые отвечают субмаксимальным классам в $P_{3}$ ). Кроме того, в решетке $\mathcal{L}_{3}$ выделены довольно крупные подрешетки, которые определяются $S$-классификацией функций трехзначной логики $[8,13]$ (48 элементов) и параметрической классификацией $[1,2]$ (2986 элементов). Отметим также работу [9], где описаны все 144 дискриминаторных класса в $P_{3}$ (классы, содержащие тернарный

* Место работы: МГУ им. М. В. Ломоносова, e-mail: ssmarchen@yandex.ru 
дискриминатор $p$ ), и работу [12], где найдены все 1504 замкнутых класса в $P_{3}$, которые являются пересечениями предполных классов. Имеются и другие результаты, связанные с решеткой $\mathcal{L}_{3}$.

В работе [6] полностью решен вопрос о числе замкнутых классов в $P_{k}$, содержащих нетривиальные однородные функции. Из результатов работы [6] следует, в частности, что в $P_{3}$ имеется лишь конечное число замкнутых классов, содержащих существенно многоместные (зависящие по крайней мере от двух переменных) линейные функции. Описать все такие замкнутые классы в [6] не удалось, хотя была получена верхняя оценка 9 для порядков данных классов. Поскольку линейные функции - важный тип функций трехзначной логики, представляется интересной задача об описании всех замкнутых классов в $P_{3}$, которые содержат существенно многоместные динейные функции.

В данной статье полностью решена эта задача. В теореме 2 перечислены все 23 замкнутых класса, которые подчиняются сформулированному условию. Приведена верхняя оценка 3 для порядков таких классов, эта оценка достижима.

\section{2. Основные понятия}

Пусть $E_{3}=\{0,1,2\}, P_{3}-$ множество всех функций на $E_{3}$ (множество функций трехзначной логики). На множестве $E_{3}$ рассматриваем функцию $2 x+2 y$ (сложение и умножение здесь и далее проводятся по модулю 3).

Для любого $i$ из $E_{3}$ обозначим через $T_{i}$ множество всех функций $f$ из $P_{3}$, которые сохраняют константу $i$, т.е. удовлетворяют равенству $f(i, \ldots, i)=i$.

Пусть $\pi$ - перестановка на $E_{3}, \pi^{-1}-$ перестановка, обратная к $\pi$, и $f\left(x_{1}, \ldots, x_{n}\right) \in P_{3}$. Функция

$$
f^{\pi}\left(x_{1}, \ldots, x_{n}\right)=\pi^{-1}\left(f\left(\pi\left(x_{1}\right), \ldots, \pi\left(x_{n}\right)\right)\right)
$$

называется сопряженной с функцией $f$ относительно перестановки $\pi$. Если $f=f^{\pi}$, то функция $f$ называется самосопряженной относительно перестановки $\pi$. Множество всех функций из $P_{3}$, самосопряженных относительно перестановки $\pi$, обозначим через $T_{\pi}$. Через $H_{3}$ обозначим пересечение всех множеств $T_{\pi}$. Функции из $H_{3}$ носят название однородных функций. Отметим, что функция $2 x+2 y$ однородна.

На множестве $P_{3}$ предполагаем заданной операцию суперпозиции [14]. Замыкание множества функций $R$ обозначаем посредством $[R]$. Множества, замкнутые относительно операции суперпозиции, называем замкнутыми классами. Легко видеть, что множества $T_{i}$ и $T_{\pi}$ являются замкнутыми классами. Понятия базиса и порядка замкнутого класса предполагаем известными [14].

Пусть $L-$ класс всех функций из $P_{3}$, линейных над полем вычетов по модулю 3. Положим

$$
L_{0}=L \cap T_{0}, \quad L_{1}=L \cap T_{1}, \quad L_{2}=L \cap T_{2}, \quad L_{x+1}=L \cap T_{x+1}, \quad L H=L \cap H_{3} .
$$

Нетрудно убедиться, что каждый из перечисленных выше классов целиком включает класс $L H$ линейных однородных функций.

Следующая лемма легко доказывается перебором линейных функций от двух переменных (см. также [15]). 
Лемма 1. $В P_{3}$ имеется ровно 6 замкнутых классов линейных функиий, которые содержат существенно многоместные линейные функиии (в квадратных скобках указан базис класса):

$$
\begin{gathered}
L=[1, x+y], \quad L_{0}=[x+y], \quad L_{1}=[x+y+2], \quad L_{2}=[x+y+1], \\
L_{x+1}=[2 x+2 y+1], \quad L H=[2 x+2 y] .
\end{gathered}
$$

Определим на множестве $E_{3}$ функции $d$ и $p$ :

$$
\begin{aligned}
& d(x, y, z)= \begin{cases}x, & \text { если } x=y, \\
z & \text { в противном случае, }\end{cases} \\
& p(x, y, z)= \begin{cases}z, & \text { если } x=y, \\
x & \text { в противном случае. }\end{cases}
\end{aligned}
$$

Функция $d$ называется дуалъным дискриминатором, функция $p-$ тернарным дискриминатором. Отметим, что функции $d, p$ однородны.

Трехместную функцию $f$ назовем мажоритарной, если выполняются тождества

$$
f(x, x, y)=f(x, y, x)=f(y, x, x)=x .
$$

Легко видеть, что функция $d$ мажоритарна.

\section{3. Основные результаты}

Следующее утверждение доказано в [6] (лемма 5). Однако ввиду малодоступности издания [6] мы воспроизводим ниже основные моменты доказательства.

Лемма 2. Произвольный замкнутый класс из $P_{3}$, который содержит функцию $2 x+2 y$ и какую-либо нелинейную функцию, содержит также мажоритарную функиию от трех переменных.

Доказательство. Пусть $R$ - замкнутый класс указанного в лемме типа. Если нелинейная функция $f$ из $R$ зависит более чем от трех переменных, то отождествлением переменных из нее можно получить нелинейную функцию, зависящую не более чем от трех переменных (этот факт установлен, например, в [3]). Поэтому далее будем предполагать, что функция $f$ зависит от трех переменных (от некоторых, возможно, несущественным образом).

Пусть функция $f$ представима полиномом по модулю 3 ,

$$
f(x, y, z)=\sum_{0 \leqslant i, j, k \leqslant 2} a_{i j k} x^{i} y^{j} z^{k},
$$

где $a_{i j k} \in E_{3}$ и $x^{0}=1$. Рассмотрим сначала случай, когда любая из функций

$$
f(x, x, y), \quad f(x, y, x), \quad f(x, y, y), \quad f(x, y, 2 x+2 y)
$$

линейна. Из условия линейности первых трех функций последовательности (3) получаем следующие три системы линейных уравнений: 


$$
\begin{aligned}
a_{222}+a_{112}+a_{202}+a_{022} & =0, \\
a_{221}+a_{111}+a_{201}+a_{021} & =0, \\
a_{212}+a_{122}+a_{102}+a_{012} & =0, \\
a_{211}+a_{121}+a_{101}+a_{011} & =0, \\
a_{220}+a_{110}+a_{200}+a_{020} & =0, \\
a_{002} & =0, \\
a_{222}+a_{121}+a_{220}+a_{022} & =0, \\
a_{212}+a_{111}+a_{210}+a_{012} & =0, \\
a_{221}+a_{122}+a_{120}+a_{021} & =0, \\
a_{211}+a_{112}+a_{110}+a_{011} & =0, \\
a_{202}+a_{101}+a_{200}+a_{002} & =0, \\
a_{020} & =0, \\
a_{222}+a_{211}+a_{220}+a_{202} & =0, \\
a_{122}+a_{111}+a_{120}+a_{102} & =0, \\
a_{221}+a_{212}+a_{210}+a_{201} & =0, \\
a_{121}+a_{112}+a_{110}+a_{101} & =0, \\
a_{022}+a_{011}+a_{020}+a_{002} & =0, \\
a_{200} & =0 .
\end{aligned}
$$

Воспользовавшись представлением (2) и полученными равенствами $a_{002}=a_{020}=$ $a_{200}=0$, выпишем для функции $f(x, y, 2 x+2 y)$ сумму нелинейных слагаемых и затем применим условие линейности функции $f(x, y, 2 x+2 y)$. Получим систему линейных уравнений

$$
\begin{aligned}
2 a_{222}+2 a_{211}+2 a_{121}+2 a_{112}+a_{220}+a_{202}+a_{022} & =0 \\
2 a_{221}+2 a_{212}+2 a_{122}+2 a_{111}+a_{210}+2 a_{201}+2 a_{102}+a_{012} & =0 \\
2 a_{221}+2 a_{212}+2 a_{122}+2 a_{111}+a_{120}+a_{102}+2 a_{021}+2 a_{012} & =0 \\
2 a_{222}+2 a_{211}+2 a_{121}+2 a_{112}+2 a_{202}+2 a_{022}+a_{110}+2 a_{101}+2 a_{011} & =0 \\
a_{202}+2 a_{101} & =0 \\
a_{022}+2 a_{011} & =0
\end{aligned}
$$

Решая систему уравнений (4)-(7), приходим к равенствам

$$
\begin{aligned}
& a_{222}=a_{221}=a_{212}=a_{122}=a_{211}=a_{121}=a_{112}=a_{220}=a_{202}=a_{022}=a_{110}= \\
& a_{101}=a_{011}=0, \quad a_{120}=a_{111}+2 a_{210}, \quad a_{201}=2 a_{210}, \quad a_{102}=a_{111}+a_{210}, \\
& a_{021}=2 a_{111}+a_{210}, \quad a_{012}=2 a_{111}+2 a_{210} .
\end{aligned}
$$

Таким образом, полагая

$$
a=a_{111}, \quad b=a_{210}, \quad c_{1}=a_{100}, \quad c_{2}=a_{010}, \quad c_{3}=a_{001}, \quad c_{4}=a_{000},
$$

получаем следующее полиномиальное представление функции $f$ :

$$
\begin{aligned}
& f(x, y, z)= \\
& a x y z+b x^{2} y+(a+2 b) x y^{2}+2 b x^{2} z+(a+b) x z^{2}+(2 a+b) y^{2} z+ \\
& 2(a+b) y z^{2}+c_{1} x+c_{2} y+c_{3} z+c_{4},
\end{aligned}
$$

где в силу нелинейности функции $f$ коэффициенты $a, b$ не равны одновременно нулю. 
Если $c_{1}+c_{2}+c_{3}=2 a+1$ и $c_{4}=0$, то, как показано в работе [3], суперпозициями функций $f, 2 x+2 y$ можно получить мажоритарную функцию $d$.

Предположим, что $c_{1}+c_{2}+c_{3} \neq 2 a+1$ и $c_{4}=0$. Тогда $f(x, x, x) \in\{0,2 x\}$. В случае функции 0 получаем

$$
x+y=2 f(x, x, x)+2(2 x+2 y),
$$

а в случае функции $2 x-$

$$
x+y=2(2 x+2 y) .
$$

Имея в классе $R$ функцию $x+y$, сводим рассматриваемый случай к предыдущему, добавляя к функции $f(x, y, z)$ необходимую линейную часть.

Пусть $c_{1}+c_{2}+c_{3} \neq 2 a+1$ и $c_{4} \neq 0$. Тогда $f(x, x, x) \in\left\{c_{4}, 2 x+c_{4}\right\}$. В случае константы $c_{4}$ получаем

$$
x+y+2 c_{4}=2(2 x+2 y)+2 f(x, x, x) .
$$

Значит, функция $f(x, y, z)+y+2 c_{4}$ имеет ту же нелинейную часть, что и функция $f(x, y, z)$, однако ее свободный член равен 0 . Поэтому приходим к одному из разобранных выше случаев. Если же $f(x, x, x)=2 x+c_{4}$, то замечаем, что функция $2 f(x, y, z)+c_{4}$ сохраняет 0. Кроме того, очевидно, отождествление в ней любых двух переменных приводит к линейной функции.

Продолжая доказательство, предположим, что среди функций ряда (3) имеется нелинейная функция. Обозначим ее через $g(x, y)$. Представим функцию $g$ полиномом по модулю 3:

$$
g(x, y)=\sum_{0 \leqslant i, j \leqslant 2} a_{i j} x^{i} y^{j}
$$

где хотя бы один из коэффициентов $a_{22}, a_{21}, a_{12}, a_{11}, a_{20}, a_{02}$ отличен от 0 .

Рассмотрим сначала случай, когда $g(x, x)=x$. Это равенство дает следующие условия на коэффициенты полинома функции $g$ :

$$
\begin{gathered}
a_{22}+a_{11}+a_{20}+a_{02}=0, \\
a_{21}+a_{12}+a_{10}+a_{01}=1, \\
a_{00}=0 .
\end{gathered}
$$

Предположим, что $a_{22} \neq 0$. Непосредственной проверкой с учетом равенств (8)-(10) убеждаемся в справедливости следующих соотношений:

$$
\begin{aligned}
& g(x, 2 x+2 y)=a_{22} x^{2} y^{2}+2\left(a_{21}+a_{12}\right) x^{2} y+a_{12} x y^{2}+a_{20} x y+a_{11} x^{2}+ \\
& a_{02} y^{2}+\left(a_{21}+a_{01}+1\right) x+2 a_{01} y, \\
& g(2 x+2 y, y)=a_{22} x^{2} y^{2}+a_{21} x^{2} y+2\left(a_{21}+a_{12}\right) x y^{2}+a_{02} x y+a_{20} x^{2}+ \\
& a_{11} y^{2}+2 a_{10} x+\left(a_{12}+a_{10}+1\right) y .
\end{aligned}
$$

Поскольку $x_{1}+x_{2}+x_{3}+x_{4} \in[2 x+2 y]$, в класс $R$ входит также функция

$$
\begin{aligned}
& g_{1}(x, y)=g(x, y)+g(x, 2 x+2 y)+g(2 x+2 y, y)+x= \\
& \left(a_{21}+2 a_{12}\right) x^{2} y+\left(2 a_{21}+a_{12}\right) x y^{2}+2 a_{22} x y+\left(a_{11}+2 a_{20}\right) x^{2}+ \\
& \left(a_{11}+2 a_{02}\right) y^{2}+\left(a_{21}+a_{01}+2\right) x+\left(a_{12}+a_{10}+1\right) y
\end{aligned}
$$


(учитываем при этом равенство (8)). Далее, с использованием функции $2 x_{1}+x_{2}+x_{3}$ из класса $[2 x+2 y]$ получаем, что классу $R$ принадлежит функция

$$
g_{2}(x, y)=g_{1}(x, y)+g_{1}(y, x)+2 x=a_{22} x y+a_{22} x^{2}+a_{22} y^{2}+y
$$

(учитываем равенства (8) и (9)).

Функция $g_{2}(x, y)$ совпадает с одной из функций

$$
x y+x^{2}+y^{2}+y, \quad 2 x y+2 x^{2}+2 y^{2}+y .
$$

Однако первая из этих функций получается из второй по формуле $2 g_{2}(x, y)+2 y$. Поэтому далее ограничимся рассмотрением только первой функции $g_{2}$. Проверяем, что она является самосопряженной относительно перестановки $x+1$ и выполняются равенства $g_{2}(0,1)=2, g_{2}(1,0)=1$. Поэтому функция $g_{2}$ не является однородной. Согласно работе [11] функции $g_{2}, 2 x+2 y$ образуют полную систему в классе функций, самосопряженных относительно перестановки $x+1$. Этому классу принадлежит, в частности, мажоритарная функция $d$.

Пусть теперь $a_{22}=0$. Определим в классе $R$ функцию $g_{3}$ :

$$
\begin{aligned}
& g_{3}(x, y)=g(x, y)+g(y, x)+x+y= \\
& \left(a_{21}+a_{12}\right) x^{2} y+\left(a_{21}+a_{12}\right) x y^{2}+\left(a_{20}+a_{02}\right) x y+\left(a_{20}+a_{02}\right) x^{2}+ \\
& \left(a_{20}+a_{02}\right) y^{2}+\left(a_{10}+a_{01}+1\right) x+\left(a_{10}+a_{01}+1\right) y .
\end{aligned}
$$

Предположим сначала, что функция $g_{3}$ линейна, т.е.

$$
a_{21}+a_{12}=a_{20}+a_{02}=0 .
$$

Тогда ввиду равенств (8) и (11) функция $g(x, y)$ приобретает вид

$$
a_{21} x^{2} y+2 a_{21} x y^{2}+a_{20} x^{2}+a_{20} y^{2}+a_{10} x+a_{01} y
$$

где в силу (9) выполняется соотношение $a_{10}+a_{01}=1$. Если $a_{21}=0$, то, как нетрудно проверить, в полиноме функции $g(g(x, y), y)$ слагаемое $x^{2} y^{2}$ входит с коэффициентом $a_{20}$. Однако $a_{20} \neq 0$, поскольку в противном случае функция $g(x, y)$ была бы линейной. Следовательно, мы приходим к уже рассмотренному случаю.

Пусть $a_{21} \neq 0$. Имеем тогда

$$
g(x, 2 x+2 y)=2 a_{21} x^{2} y+a_{20} x y+2 a_{20} y^{2}+\left(a_{21}+a_{01}+1\right) x+2 a_{01} y .
$$

Значит, классу $R$ принадлежит функция

$$
\begin{aligned}
& g_{4}(x, y)=g(x, 2 x+2 y)+g(y, 2 x+2 y)+x+y= \\
& 2 a_{21} x^{2} y+2 a_{21} x y^{2}+2 a_{20} x y+2 a_{20} x^{2}+2 a_{20} y^{2}+\left(a_{21}+2\right) x+\left(a_{21}+2\right) y .
\end{aligned}
$$

Если $a_{20}=0$, то

$$
g_{4}(x, y) \in\left\{x^{2} y+x y^{2}+x+y, \quad 2 x^{2} y+2 x y^{2}\right\} .
$$

Каждая из этих функций получается из другой по формуле

$$
2 g_{4}(x, y)+x+y \text {. }
$$

Поэтому будем считать, что имеются обе функции $g_{4}$. Обозначим первую из них через $g_{4}^{\vee}$, вторую - через $g_{4}^{\&}$. Функции $g_{4}^{\vee}, g_{4}^{\&}$ имеют векторы значений (012110202) 
и (000010002) (при лексикографическом упорядочении наборов аргументов). Положим

$$
m(x, y, z)=g_{4}^{\vee}\left(g_{4}^{\vee}\left(g_{4}^{\&}(x, y), g_{4}^{\&}(x, z)\right), g_{4}^{\&}(y, z)\right) .
$$

$\mathrm{C}$ помощью векторов значений убеждаемся, что имеют место тождества

$$
m(x, x, y)=m(x, y, x)=m(y, x, x)=x,
$$

т.е. $m(x, y, z)$ - мажоритарная функция.

Пусть $a_{21}=a_{20}$. Вновь имеем две функции $g_{4}$, каждая из которых получается из другой по формуле (12). Эти функции задаются векторами значений (022212222) и (020211012). Как и выше, определяем через них функцию по формуле (13), которая оказывается мажоритарной.

Наконец, при $a_{21} \neq a_{20}, a_{20} \neq 0$ имеем также две функции $g_{4}$, каждая из которых поолучается из другой по формуле (12). Эти функции задаются векторами (01111112) и (001012122). Вновь по формуле (13) определяем в классе $R$ мажоритарную функцию $m(x, y, z)$.

Итак, если функция $g_{3}$ линейна, то в классе $R$ имеется мажоритарная функция от трех переменных. Предположим теперь, что функция $g_{3}$ нелинейна, т.е. хотя бы одна из сумм $a_{21}+a_{12}, a_{20}+a_{02}$ отлична от нуля. Однако в случае $a_{21}+a_{12}=0$ мы, как и выше, получаем нелинейную функцию $g_{3}\left(g_{3}(x, y), y\right)$, в полином которой входит слагаемое $x^{2} y^{2}$ с ненулевым коэффициентом. Если же $a_{21}+a_{12} \neq 0$, то в силу равенства (9) имеем $a_{10}+a_{01}+1=2\left(a_{21}+a_{12}+1\right)$. Поэтому данный случай совпадает с рассмотренным случаем функции $g_{4}$.

Для завершения доказательства леммы остается рассмотреть случай, когда $g(x, x) \neq x$. Если $g_{0}(x)=g(x, x)$ и $g_{0}(x)$ - константа, то функция

$$
h(x, y)=2 g_{0}(x)+x+g(x, y)
$$

из класса $R$, очевидно, нелинейна и удовлетворяет равенству $h(x, x)=x$. Если функция $g_{0}$ линейна и отлична от константы, то $g_{0}(x)$ есть перестановка на $E_{3}$. Следовательно, одна из функций

$$
h(x, y)=g_{0}(g(x, y)), \quad h(x, y)=g_{0}\left(g_{0}(g(x, y))\right)
$$

будет нелинейной функцией, удовлетворяющей условию $h(x, x)=x$.

Предположим, наконец, что функция $g_{0}(x)$ нелинейна, Тогда функция

$$
h(x, y)=2 g_{0}(2 x+2 y)+g_{0}(x)+x
$$

нелинейна и удовлетворяет условию $h(x, x)=x$. Лемма доказана.

В дальнейшем мы хотим в условиях леммы 2 иметь не произвольную мажоритарную функцию от трех переменных, а конкретную мажоритарную функцию $d$. С этой целью обратимся к работе [17]. В ней, в частности, установлено, что произвольный замкнутый класс из $P_{3}$, содержащий мажоритарную функцию от трех переменных, содержит либо функцию $d$, либо функцию, сопряженную с одной из мажоритарных функций $m_{0}(x, y, z), m_{109}(x, y, z)$ (обозначения взяты из работы [17]). Будучи мажоритарными, функции $m_{0}$ и $m_{109}$ полностью определяются данным условием на всех наборах из $E_{3}^{3}$, содержащих не более двух различных значений. На последовательности наборов

$$
(0,1,2),(0,2,1),(1,0,2),(1,2,0),(2,0,1),(2,1,0)
$$


функции $m_{0}$ и $m_{109}$ задаются соответственно векторами значений (000000) и (011001).

Лемма 3. Функиия $d$ входит в каждое из множеств $\left[m_{0}, 2 x+2 y\right],\left[m_{109}, 2 x+2 y\right]$.

Доказательство. Начнем с функции $m_{0}$. Положим

$$
f_{1}(x, y, z)=2 m_{0}(x, y, z)+y+z
$$

Проверяем, что на последовательности наборов (14) функция $f_{1}$ дает вектор значений (002211) и, кроме того, выполняются тождества

$$
f_{1}(x, x, y)=f_{1}(x, y, x)=f_{1}(x, y, y)=y
$$

Положим далее

$$
f_{2}(x, y, z)=f_{1}\left(f_{1}(x, y, z), y, z\right) .
$$

Пользуясь приведенными выше соотношениями для функции $f_{1}$, убеждаемся, что на последовательности (14) функция $f_{2}$ имеет вектор значений (002211), а значения $f_{2}$ на остальных наборах подчиняются равенствам

$$
f_{2}(x, x, y)=f_{2}(x, y, x)=x, \quad f_{2}(x, y, y)=y \text {. }
$$

Таким образом, $f_{2}$ - мажоритарная функция.

Образуем теперь функцию

$$
f_{3}(x, y, z)=2 m_{0}(x, y, z)+2 f_{2}(x, y, z)
$$

Легко видеть, что $f_{3}$ - мажоритарная функция. Кроме того, на последовательности (14) функция $f_{3}$ дает вектор значений (001122), т.е. на последовательности (14) значения функции $f_{3}(x, y, z)$ совпадают со значениями переменной $x$. Иначе говоря, функция $f_{3}$ задается соотношениями

$$
f_{3}(x, y, z)= \begin{cases}y, & \text { если } y=z, \\ x & \text { в противном случае }\end{cases}
$$

Эти соотношения показывают, что функция $d$ получается из функции $f_{3}$ перестановкой переменных.

Обратимся теперь к функции $m_{109}$. Пусть

$$
m_{2}(x, y, z)=2 m_{109}(x, y, z)+2 m_{109}(x, z, y) .
$$

Тогда $m_{2}$ - мажоритарная функция с вектором значений (222222) на последовательности (14). Это означает, что функция $m_{2}$ сопряжена с функцией $m_{0}$ (посредством, например, перестановки $x+1)$. Используя очевидный «принцип сопряженности» (если функция $g$ реализуется формулой над множеством функций $G$, то сопряженная функция $g^{*}$ реализуется той же формулой, но над множеством сопряженных функций $\left.G^{*}\right)$, получаем, что формула, реализующая функцию $d$ над множеством функций $\left\{m_{0}, 2 x+2 y\right\}$, будет реализовывать ту же функцию $d$ над множеством $\left\{m_{2}, 2 x+2 y\right\}$. Здесь мы пользуемся тем, что функция $d$ является самосопряженной относительно любых перестановок. Лемма доказана. 
Теорема 1. Произвольный замкнутый класс из $P_{3}$, содержащий функцию $2 x+2 y$ и какую-либо нелинейную функцию, совпадает с одним из следующих 17 классов:

$$
\begin{gathered}
P_{3}, \quad T_{0}, \quad T_{1}, \quad T_{2}, \quad T_{0} \cap T_{1}, \quad T_{0} \cap T_{2}, \quad T_{1} \cap T_{2}, \quad T_{0} \cap T_{1} \cap T_{2}, \quad T_{x+1}, \\
T_{x+1} \cap T_{0}, \quad T_{2 x}, \quad T_{2 x} \cap T_{1}, \quad T_{2 x+1}, \quad T_{2 x+1} \cap T_{0}, \quad T_{2 x+2}, \quad T_{2 x+2} \cap T_{0}, \quad H_{3} .
\end{gathered}
$$

Доказательство. Как установлено выше, произвольный замкнутый класс $R$ рассматриваемого в теореме типа содержит дуальный дискриминатор $d$. Известно [3], что функции $d, 2 x+2 y$ образуют базис класса $H_{3}$. Таким образом, перечисление всех классов $R$, удовлетворяющих условию теоремы, равносильно перечислению всех замкнутых классов, целиком включающих класс $H_{3}$. Однако классу $H_{3}$ принадлежит, в частности, тернарный дискриминатор $p$. В работе [9] найдены все 144 замкнутых класса, содержащих дискриминатор $p$. Каждый из них охарактеризован в терминах сохранения некоторого (конечного) множества предикатов. Если отобрать из этих предикатов только те предикаты, которые сохраняются функцией $2 x+2 y$, то в результате получатся множества предикатов, определяющие в точности классы последовательности (15). Теорема доказана.

Теорема 2. $В P_{3}$ имеется ровно 23 замкнутых класса, которые содержат существенно многоместные линейные функиии: 6 классов (1), которые состоят только из линейных функций, и 17 классов (15), включающих нелинейные функции.

В работе [10] доказано, что порядок произвольного дискриминаторного класса из $P_{3}$ не превосходит 3 . В [3] установлено, что порядок класса $H_{3}$ равен 3 . Легко видеть, что порядки всех классов (1) равны 2. Можно показать, что порядки всех классов из (15), отличных от класса $H_{3}$, также равны 2.

В связи с полученными результатами возникает вопрос: можно ли обобщить теорему 2 на (некоторые) одноместные линейные функции? Отрицательный отовет на этот вопрос вытекает из работ $[3,5]$ : для всякой одноместной линейной функиии $f$ из $P_{3}$ существует континуальное число замкнутых классов, содержащих функцию $f$.

\section{Список литературы}

1. Данильченко, А. Ф., "О параметрической выразимости функций трехзначной логики", Алгебра и логика, 16:4 (1977), 397-416.

2. Данильченко, А. Ф., "Параметрически замкнутые классы функций трехзначной логики", Известия АН МССР, 2 (1979), 13-20.

3. Марченков, С. С., "О замкнутых классах самодвойственных функций многозначной логики", Проблемы кибернетики, 36 (1979), 5-22.

4. Марченков, С. С., "Однородные алгебры”, Проблемы кибернетики, 39 (1982), 85-106.

5. Марченков, С. С., "О замкнутых классах самодвойственных функций многозначной логики II", Проблемы кибернетики, 40 (1983), 261-266.

6. Марченков, С. С., "О замкнутых классах в $P_{k}$, содержащих однородные функции", Препринт ИПМ им. М. В. Келдыша АН СССР, 1984, № 35, 1-28.

7. Марченков, С. С., "Клоновая классификация дуально дискриминаторных алгебр с конечным носителем", Математические заметки, 61:3 (1997), 359-366.

8. Марченков С. С., S-классификация функций трехзначной логики, Физматлит, Москва, 2001, $79 \mathrm{c}$.

9. Марченков, С. С., “Дискриминаторные классы трехзначной логики”, Математические вопросы кибернетики, 12 (2003), 15-26. 
10. Марченков, С. С., "О порядках дискриминаторных классов многозначной логики”, $M a-$ тематические заметки, 86:4 (2009), 550-556.

11. Марченков, С. С., Деметрович, Я., Ханнак, Я., "О замкнутых классах самодвойственных функций в $P_{3}$ ", Методы дискретного анализа в решении комбинаторных задач, 1980, № 34, 38-73.

12. Нагорный, А. С., "О распределении трехзначных функций по предполным классам", Вестник Московского ун-та. Серия 15. Вычисл. матем. и кибернетика, 2012, №3, $45-52$.

13. Нгуен Ван Хоа, "О структуре самодвойственных замкнутых классов трехзначной логики $P_{3}$ ", Дискретная математика, 4:4 (1992), 82-95.

14. Яблонский С. В., Введение в дискретную математику, Наука, Москва, 1986, 384 с.

15. Bagynszki, J., Demetrovics, J., "The lattice of linear classes in prime-valued logics", Banach Center Publ., 7 (1982), 105-123.

16. Baker, A., Pixley, A. F., "Polynomial interpolation and the Chinese Remainder Theorem for algebraic systems", Math. Zeitschrift, 143 (1975), 165-174.

17. Csakany, B., "All minimal clones on three-element set", Acta Cybernet., 6:3 (1983), 227-238.

18. Lau, D., "Bestimmung der Ordnung maximaler Klassen von Funktionen der $k$-vertigen Logik", Z. Math. Logik Grundlag. Math., 24:1 (1978), 79-96.

Статья поступила 14.05.2014. 